Stefano Vidussi

\title{
On the number of components of the symplectic representatives of the canonical class
}

Received March 8, 2006 and in revised form May 13, 2006

\begin{abstract}
We show that there exists a family of simply connected, symplectic 4-manifolds such that the (Poincare dual of the) canonical class admits both connected and disconnected symplectic representatives. This answers a question raised by Fintushel and Stern.
\end{abstract}

Keywords. Symplectic 4-manifolds, canonical class

\section{Introduction and statement of the result}

An important result of 4-dimensional symplectic topology, proven first by Taubes in [T] using the relation between Seiberg-Witten and Gromov invariants and then (under minor assumptions) by Donaldson and Smith in [DS] via Lefschetz fibration techniques, is the existence of a symplectic representative of the (Poincaré dual of the) canonical class of a symplectic 4 -manifold with $b_{+}>1$. These proofs, in general, do not provide a sufficiently explicit construction of such a representative, nor make any statement concerning uniqueness, number of components, or their genus. It is therefore a non-obvious task, given a symplectic 4-manifold, to provide explicitly such a representative.

An interesting case of this problem is described in [FS4]; Fintushel and Stern show that, for any choice of positive integers $\left\{\left(g_{i}, m_{i}\right): i=1, \ldots, n\right\}$, there exists a (minimal) simply connected symplectic manifold $X$ whose canonical class $K_{X} \in H_{2}(X, \mathbb{Z})$ is represented by an embedded symplectic surface $\Sigma$ with $\sum_{i=1}^{n} m_{i}$ connected components:

$$
\Sigma=\coprod_{i=1}^{n} \coprod_{j=1}^{m_{i}} \Sigma_{g_{i}, j} \in K_{X},
$$

where $\Sigma_{g_{i}, j}$ is a connected surface of genus $g_{i}$. These manifolds are obtained through natural symplectic operations, i.e. symplectic fiber sum and symplectic rational blowdown, on simply connected elliptic surfaces without multiple fibers $E(s)$. The representative $\Sigma$ of (1.1) is, in some sense, the natural result of such operations when we start with the algebraic representative of the canonical class of the elliptic surface, namely the disjoint union of $s-2$ copies of the fiber.

S. Vidussi: Department of Mathematics, University of California, Riverside, CA 92521, USA; e-mail: svidussi@math.ucr.edu

Mathematics Subject Classification (2000): Primary 57R17 
Led by this construction, Fintushel and Stern ask whether, for a symplectic manifold whose canonical class admits a symplectic representative as in (1.1), the set of integers $\left\{\left(g_{i}, m_{i}\right): i=1, \ldots, n\right\}$ is a symplectic invariant. This question is carefully asked under the hypothesis of all $g_{i} \geq 2$; without this constraint, plenty of counterexamples can be found in [FS3], [S], [V], where it is shown (with different constructions) that the canonical class of $E(s)$, for $s \geq 4$, can be symplectically represented by a connected (non-algebraic) torus.

In this paper, we will provide an answer, in the negative, to the aforementioned question, by showing that it is possible to exhibit a connected symplectic representative for $K_{X}$ for the family of manifolds constructed in [FS4]. With obvious modifications, symplectic representatives with any number of components between 1 and $\sum_{i=1}^{n} m_{i}$ can be obtained.

Roughly speaking, the idea behind our construction consists in "sewing together" some (or all) the components of the symplectic representative of (1.1), while keeping the resulting representative symplectic. For the manifolds $X$ considered in this paper such an internal surgery is explicitly exhibited, and similar cases can be treated analogously. However, it is conceivable that a similar process exists in general, whenever a disconnected representative is available: we are not aware, at this point, of obstructions to the existence of a connected symplectic representative of the canonical class.

We want to point out that, without contradiction, the result we obtain does not exclude the use of a numerical symplectic invariant related to the number of components of symplectic representatives of the canonical class (the set of integers $\left\{\left(g_{i}, m_{i}\right): i=1, \ldots, n\right\}$ for a maximal number of components $\sum_{i=1}^{n} m_{i}$ could be such an example); it just stresses the need of accounting for the various representatives. In particular it is possible that the set of integers $\left\{\left(g_{i}, m_{i}\right), i=1, \ldots, n\right\}$ determined in [FS4] is a symplectic invariant of the family of manifolds defined therein.

Organization of the paper. Sections 2 and 3 provide some preliminary material that will be of use in Section 4 for our main construction. More precisely, in Section 2 we discuss a presentation of the elliptic surfaces $E(n), n \geq 2$, as symplectic link surgery manifolds, as the first step in identifying some natural submanifolds. In Section 3 we exhibit various symplectic spheres and tori in $E(n)$ that will be the building blocks of our construction. Section 4 contains an inductive presentation of the manifold $X$, reviewing some of the steps of [FS4], which leads to the construction of the disconnected and connected symplectic representatives of $K_{X}$.

\section{Elliptic surfaces as link surgery manifolds}

The construction of the manifold $X$ in [FS4] starts by symplectic summing of elliptic surfaces along the fiber $F$ (obviously a symplectic submanifold) and along a second symplectic torus $R$ (a rim torus) that arises, in the surface $E(n+2)=E(n+1) \#_{F_{1}=F_{2}} E(1)$ $(n \geq 0)$, by identifying two tori, in the exterior of the fibers $F_{i}$, that become essential after the sum. In order to study this construction, we will present an elliptic surface $E(n+2)$ as a link surgery manifold (see [FS2] for the definition) obtained from the Hopf link. This 
presentation will help us identify some symplectic submanifolds (spheres and tori) in the elliptic surface $E(n+2)$, for $n \geq 0$, that we will use in our construction.

Consider the Hopf link $H=K_{0} \cup K_{1}$ and, for future reference, denote by $K$ the simple closed curve, in $S^{3} \backslash v H$, which links once $K_{0}$ and $K_{1}$ as in Figure 1 .

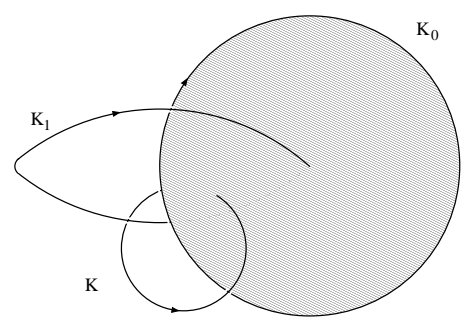

Fig. 1. The simple closed curve $K$ and the Hopf link $H$; the spanning surface of $K_{0}$ is dashed.

A more suggestive presentation of the resulting three-component link $H \cup K$ appears by considering it as the closure, with the axis originating $K_{0}$, of the braid of Figure 2 .

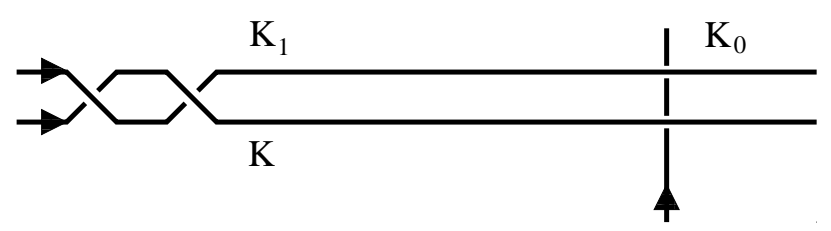

Fig. 2. The closure of the braid gives $H \cup K$.

The exterior of the link $S^{3} \backslash v H$ contains two annuli $A$ and $B$ that are fibers of two distinct fibrations having homology class (in $H^{1}\left(S^{3} \backslash v H, \mathbb{Z}\right)=\mathbb{Z}^{2}$ ) equal to $(1,0)$ and $(0,1)$ respectively. Such annuli have boundary $\lambda\left(K_{0}\right) \cup-\mu\left(K_{1}\right)$ and $-\mu\left(K_{0}\right) \cup \lambda\left(K_{1}\right)$ respectively. It is useful, for future reference, to think of $B$ as the annulus swept by an arc in the fiber $A$ of the corresponding fibration. In what follows, we will consider all the knots endowed with the framing defined by their spanning disk. Moreover, whenever we will make reference to meridians and longitudes, we will implicitly assume a specific choice of these curves is made.

Now observe that any elliptic surface $E(n+2)$ can be presented as the link surgery manifold obtained by gluing the manifold (with two boundary components) $S^{1} \times\left(S^{3} \backslash v H\right)$ to the exterior of the elliptic fiber of $E(1)$ and $E(n+1)$ : in fact $S^{1} \times\left(S^{3} \backslash v H\right)=$ $T^{2} \times\left(S^{2} \backslash v\left\{p_{0}, p_{1}\right\}\right)=T^{2} \times A$, where $A$ is the annulus defined above (removing an open neighborhood of the Hopf link from $S^{3}$ gives a circle times the annulus $A$, with the circle identified to the meridian of $K_{0}$ ). The usual fiber sum definition of elliptic surfaces can therefore be interpreted as follows:

$$
E(n+2)=\left(E(n+1) \backslash v F_{0}\right) \cup S^{1} \times\left(S^{3} \backslash v H\right) \cup\left(E(1) \backslash v F_{1}\right)
$$


where the first gluing map identifies, in the boundary 3-tori, $F_{0}$ with $S^{1} \times \mu\left(K_{0}\right)$ and (remembering that we reverse orientations) the meridian of $F_{0}$ with $-\lambda\left(K_{0}\right)$, while the second gluing map identifies $F_{1}$ with $S^{1} \times \lambda\left(K_{1}\right)$ and the meridian of $F_{1}$ with $\mu\left(K_{1}\right)$. After gluing, the fibers $F_{0}$ and $F_{1}$ get identified. Note moreover that the smooth structure of the resulting manifold is unaffected by the choice of the diffeomorphism between the fibers of the elliptic surfaces and $S^{1} \times \mu\left(K_{0}\right)$ and $S^{1} \times \lambda\left(K_{1}\right)$; we will later choose a particular identification.

We need to keep track of the two tori of $E(n+2)$ that are images of $S^{1} \times \mu\left(K_{0}\right)$ and $S^{1} \times \lambda\left(K_{0}\right)$ respectively. The first one, identified with the fiber $F$ of the elliptic fibration of $E(n+2)$, is clearly essential, but also the second one, which we denote by $R$, is essential in $E(n+2)$ (see e.g. [GS]). Due to its origin, we will call it the rim torus.

To analyze the symplectic submanifolds, we consider how the construction above leads to present $E(n+2)$ as a symplectic fiber sum: Perform Dehn surgery along $H \subset S^{3}$ with coefficient 0 along $K_{0}$ and coefficient $\infty$ along $K_{1}$ to get $S^{1} \times S^{2}$. Denote by $C_{0}$ and $C_{1}$ the cores of the solid tori: these are, up to isotopy, standard circles of the form $S^{1} \times\left\{p_{i}\right\}$ in the resulting $S^{1} \times S^{2}$. The manifold $S^{1} \times\left(S^{1} \times S^{2}\right)$ has a natural symplectic structure (of the form $d t \wedge \alpha+\epsilon \beta$, where $\alpha$ represents the fibration of $S^{1} \times S^{2}$ and $\beta$ is a volume form on the sphere). The tori $S^{1} \times C_{i}$ are symplectic, framed, self-intersection zero tori and, after scaling the symplectic forms on each summand if necessary, we can write

$$
E(n+2)=E(n+1) \#_{F_{0}=S^{1} \times C_{0}} S^{1} \times\left(S^{1} \times S^{2}\right) \#_{S^{1} \times C_{1}=F} E(1) .
$$

The symplectic form, away from the gluing locus, restricts to the symplectic forms of the summands.

Note that the presentation of 2.2 can be interpreted, from a certain viewpoint, as a glorified form of Weinstein's Tubular Neighborhood Theorem, in the sense that it provides information on the restriction of the symplectic form of $E(n+2)$ to the submanifold $F \times A$ which separates $E(n+1) \backslash \nu F$ and $E(1) \backslash \nu F$; application of Weinstein's Theorem to a fiber of $E(1)$, and then fiber sum with $E(n+1)$, tells us that $F \times A$, up to symplectomorphism, has product symplectic structure (where on the annulus $A$ we take the restriction of the symplectic form on the sphere). This is the same as the symplectic structure arising on the image of $S^{1} \times\left(S^{1} \times S^{2} \backslash v\left(C_{0} \sqcup C_{1}\right)\right)=F \times A$ in the fiber sum of 2.2.

\section{Some symplectic spheres and tori in $E(n+2)$}

In this section we will exploit the presentation of the elliptic surface $E(n+2)$ of Section 2 to identify some symplectically embedded spheres and tori that will be the building blocks for our (and Fintushel-Stern's) construction.

We start with the tori. Three symplectic, framed, self-intersection zero tori arise from the presentations of (2.1) and (2.2). The first is the fiber $F$. The second one, $R$, is one of the two "marked" rim tori that become nontrivial in the fiber sum of $E(n+1)$ and $E(1)$ (see Section 3.1 of [GS]), the other one being identified with the image of $\mu\left(K_{1}\right) \times$ $\lambda\left(K_{1}\right)$. This essential torus is naturally Lagrangian and becomes symplectic by a small 
perturbation of the symplectic structure, as discussed in [G]. The third one is the image of $S^{1} \times K$. Their properties are summarized in the following proposition.

Proposition 1. Consider the inclusion map

$$
S^{1} \times\left(S^{3} \backslash v H\right) \hookrightarrow E(n+2) .
$$

The following holds:

(1) The image $F$ of the torus $S^{1} \times \mu\left(K_{0}\right)$ under the inclusion above is a symplectic, framed, connected submanifold of $E(n+2)$.

(2) The image $R$ of the torus $S^{1} \times \lambda\left(K_{0}\right)$ is a Lagrangian, framed, connected submanifold of $E(n+2)$.

(3) The image $T$ of the torus $S^{1} \times K$ is a symplectic, framed, connected submanifold of $E(n+2)$ satisfying $[T]=[F]+[R] \in H_{2}(E(n+2))$.

Moreover, the three tori above can be assumed to be disjoint.

Proof. The first part of the statement clearly holds true. The nonobvious part is to prove that $R$ and $T$ are respectively Lagrangian and symplectic with respect to the symplectic structure on $E(n+2)$ induced by the symplectic fiber sum of $(2.2)$. Up to isotopy, we can assume that $\lambda\left(K_{0}\right)$ lies on a spanning disk of $K_{0}$ (see Figure 1 ); it is therefore contained in a fiber of the fibration of $S^{1} \times S^{2}$ obtained by capping off the disk fibration of $S^{3} \backslash v K_{0}$ induced by the spanning disks. As a consequence, the symplectic structure on $S^{1} \times\left(S^{1} \times S^{2}\right)$ restricts trivially to $S^{1} \times \lambda\left(K_{0}\right)$ (its tangent space is spanned by $\partial / \partial t$ and a vector tangent to the sphere) so that the torus $R$ (homologically nontrivial) is a Lagrangian submanifold of $E(n+2)$. This proves (2) above. For what concerns (3) we note that, up to isotopy, $K$ is transversal to the fibration of $S^{1} \times S^{2}$ (see Figure 2), so that the symplectic form on $S^{1} \times\left(S^{1} \times S^{2}\right)$ never vanishes on the torus $S^{1} \times K$. As this torus is symplectic in one summand, it will be symplectic in the fiber sum of 2.2. For what concerns the homology class, observe that, in the homology of $S^{3} \backslash v H$,

$$
\begin{aligned}
{[K] } & =\operatorname{lk}\left(K, K_{0}\right)\left[\mu\left(K_{0}\right)\right]+\operatorname{lk}\left(K, K_{1}\right)\left[\mu\left(K_{1}\right)\right] \\
& =\left[\mu\left(K_{0}\right)\right]+\left[\mu\left(K_{1}\right)\right] \in H_{1}\left(S^{3} \backslash v H\right) .
\end{aligned}
$$

The meridian $\mu\left(K_{1}\right)$ is homologous (actually, isotopic) to $\lambda\left(K_{0}\right)$, so the relation follows at this point from the identification of $S^{1} \times \mu\left(K_{0}\right)$ and $S^{1} \times \lambda\left(K_{0}\right)$ with $F$ and $R$ respectively. The fact that these tori are disjoint follows directly from the construction.

The output of the previous proposition, namely that the (primitive) class $[F]+[R]$ can be represented by two symplectic submanifolds, one given by the disjoint union $F \amalg R$ and the second by $T$, will be, in fact, the main tool for our construction. Note that the curve $K$ can be interpreted as the result of circle summing of the meridian $\mu\left(K_{0}\right)$ and the longitude $\lambda\left(K_{0}\right)$. The resulting operation on the tori $F$ and $R$, which produces the symplectic torus $T$ from the symplectic torus $F$ and the Lagrangian torus $R$, represents at local level the "sewing" referred to in the Introduction. 
Having dealt with tori, we will now consider spheres. Specifically, we are interested in two groups of spheres. The first group is the "sections" of elliptic nuclei, where an elliptic nucleus is the regular neighborhood of the union of a cusp fiber and a section of an elliptic fibration. The second group of spheres is those contained in a configuration that we can use for rational blowdown. Remember that this surgery consists in replacing, in a 4-manifold, a regular neighborhood of a configuration $\Gamma_{n}$ of $n-1$ spheres as in Figure 4 . the first with self-intersection $-(n+2)$ and the remaining of self-intersection -2 , with a certain rational homology ball $B_{n}$. This rational homology ball naturally embeds in the Hirzebruch surface $\mathbf{F}_{g-1}$, as the exterior of the configuration of spheres $\left(S_{+}+f\right)$ $\cup S_{-}$, where $S_{+}$(resp. $S_{-}$) is the positive (negative) section and $f$ the fiber of the sphere fibration of $\mathbf{F}_{g-1}$; see [FS1] for the details of this construction.

We will start with the first group. It is well known that an elliptic surface $E(n+2)$ contains several disjoint elliptic nuclei (see [GS] for example). We will be interested in two of them. We have the following proposition:

Proposition 2. Let $E(n+2), n \geq 0$, be an elliptic surface, with the symplectic structure inherited from the construction of Section 2. The following holds true:

(1) $E(n+2)$ contains an elliptic nucleus $N_{F}$, with symplectic fiber $F$ and symplectic sphere $S_{F}$ of self-intersection - $(n+2)$, given by a section of the elliptic fibration.

(2) $E(n+2)$ contains an elliptic nucleus $N_{R}$, with Lagrangian fiber $R$ and Lagrangian sphere $S_{R}$ of self-intersection -2 ; with a small perturbation of the symplectic structure of $E(n+2)$ we can make $R$ and $S_{R}$ symplectic.

(3) The nuclei $N_{F}$ and $N_{R}$ are disjoint, and the torus $T$ intersects their spheres in a single, transverse point.

Proof. The first nucleus $N_{F}$ arises, in the picture of 2.2, as a regular neighborhood of a cusp fiber of the elliptic fibration and its $-(n+2)$-sphere section $S_{F}$; the symplectic fiber of this nucleus is $F$, and the section $S_{F}$ arises by gluing together a disk section of $E(n+1) \backslash v F$ with a $-(n+1)$ twist rel $\partial$ in its normal bundle (for sake of brevity we will call these disks, with the usual abuse of language, $-(n+1)$-disks $)$, a $(-1)$-disk section of $E(1) \backslash \nu F$ and (for a suitable choice of the embedding in $S^{1} \times\left(S^{3} \backslash \nu H\right)=F \times A$ ) the annulus $A \subset S^{3} \backslash \nu H$ (which has boundary identified with $\lambda\left(K_{0}\right) \cup-\mu\left(K_{1}\right)$ ). The two vanishing disks that kill the generators of $\pi_{1}(F)$ can be located in $E(n+1) \backslash v F_{0}$, as $F$ is already contained in a nucleus in $E(n+1)$. The sphere $S_{F}$ is symplectic, being a connected sum of symplectic spheres in each summand of (2.2). This proves (1).

The second nucleus $N_{R}$ contains, as regular fiber, the Lagrangian rim torus $R$. The vanishing disks that kill the generators of $\pi_{1}(R)$ can be located in $E(1) \backslash \nu F_{1}$ (using a second disk section and a vanishing disk). The $(-2)$-sphere $S_{R}$ of the nucleus is obtained, in the construction of $(2.1)$, by gluing the annulus $B$ (which has boundary identified with $\left.-\mu\left(K_{0}\right) \cup \lambda\left(K_{1}\right)\right)$ embedded in $S^{1} \times\left(S^{3} \backslash \nu H\right)=F \times A$ to two $(-1)$-vanishing disks in $E(n+1) \backslash \nu F_{0}$ and $E(1) \backslash \nu F_{1}$. The annulus $B$ is Lagrangian, as we can span at each point its tangent space by a vector $v$ in $\operatorname{ker} \alpha$ and a vector $w$ that satisfies $\beta(w, \cdot)=0$, so that the symplectic form on $F \times A$ vanishes on $B$. Similarly, the vanishing disks in the elliptic surfaces can be taken to be Lagrangian thimbles of a symplectic Lefschetz fibration (see 
[ADK, Section 4], and, for a general discussion, [D]): we start with a generic pencil of cubics in $\mathbb{P}^{2}, \pi: \mathbb{P}^{2} \backslash B \rightarrow \mathbb{P}^{1}$ (where the base locus $B$ is composed of nine points) and we endow it with a symplectic connection, given by the symplectic orthogonal complement to the fiber. Then, given a path $\gamma(t)$ on $\mathbb{P}^{1}$ joining a critical value $\pi\left(p_{c}\right)=\gamma(0)$ to a regular value $\gamma(1)$ (and otherwise disjoint from the set of critical values) we can define a Lagrangian vanishing disk as the union of the vanishing cycles on the fibers lying over the path, defined by the condition that symplectic parallel transport $\mathcal{P}$ sends them to the critical point on the singular fiber, namely

$$
p_{c} \cup \bigcup_{t} V_{t}:=p_{c} \cup \bigcup_{t}\left\{u \in \pi^{-1}(\gamma(t)) \mid \lim _{\epsilon \rightarrow 0} \mathcal{P}_{\gamma \mid[\epsilon, t]} u=p_{c}\right\} .
$$

When we symplectically blow up $\mathbb{P}^{2}$ along the base locus of the pencil to get $E(1)$, we obtain from the exceptional divisors nine symplectic sections, disjoint from the Lagrangian disk. Further fiber summing, to get other elliptic surfaces, does not affect the vanishing disk. When we recover $E(n+2)$ through the fiber sum of [2.2), we choose the identification map on the two boundary tori so as to identify the boundary of the annulus $B$ with the boundaries of the vanishing disks, obtaining this way the Lagrangian (-2)-sphere $S_{R}$. The essential Lagrangian submanifolds $R$ and $S_{R}$ can be made symplectic with a small perturbation of the symplectic form, as in [FS4]. This yields (2).

For what concerns (3), note that the intersection of $A$ and $B$ in $S^{3} \backslash v H$ gets removed in $F \times A$, due to the presence of the extra $S^{1}$ factor. Moreover, thanks to the abundance of singular fibers and sections, we can choose all the disks used in the previous construction to be disjoint (see $[\overline{\mathrm{GM}}]$ ). As a result, the nuclei $N_{F}$ and $N_{R}$ are disjoint. Finally, the torus $T$ intersects $S_{F}$ and $S_{R}$ only in the interior of $F \times A$, and the intersection is a single transverse point corresponding to the intersection of $K$ with the annuli $A$ and $B$, as can be seen from Figure 1 .

Figure 3 schematizes the relation between the two nuclei and the torus $T$.

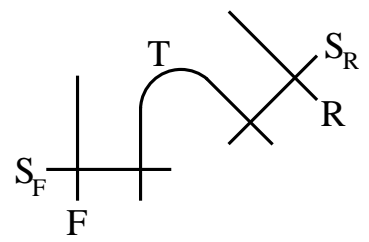

Fig. 3. Schematic representation of the relation between the two nuclei of fibers $F$ and $R$ and the torus $T$.

The second group of spheres we are interested in is the configuration of symplectically embedded spheres $\Gamma_{n}$. As discussed in [FS1] any elliptic surface $E(n)$ contains a configuration of $4 n-1$ symplectic spheres as in the diagram of Figure 4

The sphere of self-intersection $-n$ is the section of the elliptic fibration, and the configuration intersects the fiber $F$ only in $S_{F}$, with a single transverse intersection point. 


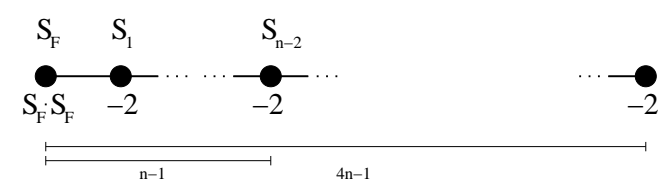

Fig. 4. The first $n-1$ spheres give the configuration $\Gamma_{n}$ in the elliptic surface $E(n+2)$.

When we fiber sum $E(2)$ to $E(n)$ we have therefore (Lemma 2.1 of [FS4]), in the resulting $E(n+2)$, a configuration of $4 n-1$ embedded symplectic spheres as in the diagram of Figure 4 (where the sphere $S_{F}$ of self-intersection $-(n+2)$, obtained by gluing two sections, is again a section of the elliptic fibration). In particular, if we keep track only of the first $n-1$ spheres of the configuration, we deduce that $E(n+2)$, for $n \geq 1$, contains the configuration $\Gamma_{n}$, whose spheres will be denoted as $S_{F}, S_{1}, \ldots, S_{n-2}$. Note that in $E(n+2)=E(2) \#_{F} E(n)$, as the nucleus determined by the rim torus $R$ is disjoint from the section of $E(2)$, it is also disjoint from the configuration; moreover the torus $T$ (entirely contained in $E(2) \backslash v F$ ) intersects the configuration in a single transverse point on the first sphere $S_{F}$.

\section{Fintushel-Stern construction and connected representatives}

For sake of clarity, we summarize some of the results discussed in the previous two sections. In the elliptic surface $E(n+2)$, for $n \geq 1$, we can identify two disjoint symplectically embedded surfaces. The first is the linear plumbing of the $n-1$ spheres $S_{F}, S_{1}, \ldots, S_{n-2}$ described in the diagram of Figure 4 The second is the nucleus $N_{R}$. The fiber $F$ intersects $S_{F}$ in a single positive point, orthogonal with respect to the symplectic structure, while it does not intersect the other spheres of $\Gamma_{n}$ nor the nucleus $N_{R}$. The symplectic torus $T$, instead, intersects both $S_{F}$ and $S_{R}$ in a single transverse point, and is otherwise disjoint from the remaining spheres and tori considered above.

In the situation above (except for the torus $T$, which has no role in their construction) Fintushel and Stern have inductively defined a family of minimal symplectic simply connected 4-manifolds whose canonical class admits a symplectic representative satisfying the condition of (1.1). We will sketch their construction and show how, by a suitable modification, we can obtain a connected representative for the canonical class. (In what follows we will reserve the symbol $\hat{\imath}$ to denote connected surfaces.)

First, for $g \geq 2$, let $X_{g}$ be the manifold obtained by symplectic rational blowdown of the configuration $\Gamma_{g}$ in $E(g+2)$, i.e. replacing $\nu \Gamma_{g}$ with the rational homology ball $B_{g} \subset \mathbf{F}_{g-1}$ (see Section 3 ). The resulting manifold is simply connected and symplectic, where the symplectic structure is obtained by grafting to $E(g+2) \backslash \nu \Gamma_{g}$ the symplectic structure induced by the embedding of $B_{g}$ in the Hirzebruch surface $\mathbf{F}_{g-1}$ endowed with a suitable symplectic structure (see $[\overline{S y} \mid)$. A symplectic representative of the canonical class $K_{X_{g}}$ (image of $K_{E(g+2)}=g[F]$ under the blowdown map) is then given by gluing $\left(F_{1} \amalg \cdots \sqcup F_{g}\right) \backslash \nu \Gamma_{g}$ (a collection of $g$ copies of the fiber with a hole) to $S_{+} \cap B_{g}$ (a sphere with $g$ holes, as $S_{+}$and $\left(S_{+}+f\right) \cup S_{-}$intersect in $g$ points). The result of this surgery 
is therefore a connected, embedded, symplectic surface of genus $g$ that we will denote by $\hat{\Sigma}_{g}$, and which represents $K_{X_{g}}$.

There is a homology class we want to keep track of, and which represents the image, under the blowdown, of the class $g[F]+[R]$. The nucleus $N_{R}$ is disjoint from $\Gamma_{g}$, so that it survives the blowdown process; we index with an $R_{X}$ its image. (A more correct, but notationally heavier, index would be $R_{X_{g}}$.) The image of the class $g[F]+[R]$ is therefore well defined and is given by $\left[\hat{\Sigma}_{g}\right]+\left[R_{X}\right]=K_{X_{g}}+\left[R_{X}\right]$. The disjoint union $\hat{\Sigma}_{g} \amalg R_{X}$ is a symplectic representative, with two connected components of genus $g$ and 1 , but (as $[T]=[F]+[R]$ ) we can represent it as well by the connected, symplectic, genus $g$ surface $\hat{\Sigma}_{g}^{R_{X}}$ obtained by gluing, much as above, $\left(F_{1} \amalg \cdots \amalg F_{g-1} \amalg T\right) \backslash \nu \Gamma_{g}$ (a collection of $g$ disjoint tori with a hole) to $S_{+} \cap B_{g}$. The surface $\hat{\Sigma}_{g}^{R_{X}}$ intersects the (-2)-sphere $S_{R_{X}}$ in a single positive transverse point, which is the image of the intersection point of $T$ and $S_{R_{X}}$ under the blowdown.

Summing up, we have a symplectic manifold $X_{g}$ which has a canonical class $K_{X_{g}}$ represented by $\hat{\Sigma}_{g}$, a connected symplectic surface of genus $g$. This manifold contains an embedded symplectic nucleus $N_{R_{X}}$ (with fiber $R_{X}$ ) disjoint from $\hat{\Sigma}_{g}$; moreover the class $K_{X_{g}}+\left[R_{X}\right]$ can be represented by the disjoint union $\hat{\Sigma}_{g} \amalg R_{X}$ or by a connected symplectic surface $\hat{\Sigma}_{g}^{R_{X}}$ of genus $g$.

The manifold $X_{g}$ is the initial step in the inductive construction of the family of manifolds of [FS4], and now we will proceed to the inductive step. In practical terms, we need to go over the proof of Lemma 2.2 of [FS4] and ensure that there is room to use, at each inductive step, a torus of the type $T$ to "sew" the components of the disconnected representative identified by Fintushel and Stern. The argument is quite straightforward but rather long to present.

Lemma 3. Let $\left\{g_{i}: i=1, \ldots, m\right\}$ be a collection of integers $\geq 2$. Let $X$ be a symplectic simply connected 4-manifold satisfying the following conditions:

(1) The canonical class $K_{X}$ of $X$ can be represented by the union $\Sigma_{g_{1}, \ldots, g_{m}}$ of $m$ disjoint connected symplectic surfaces of genus $g_{1}, \ldots, g_{m}$ or by a connected symplectic surface $\hat{\Sigma}_{g_{1}, \ldots, g_{m}}$ of genus $\sum_{i=1}^{m} g_{i}-m+1$.

(2) $X$ contains a symplectic nucleus $N_{R_{X}}$ with fiber $R_{X}$ and section $S_{R_{X}}$ disjoint from both $\Sigma_{g_{1}, \ldots, g_{m}}$ and $\hat{\Sigma}_{g_{1}, \ldots, g_{m}}$. The class $K_{X}+\left[R_{X}\right]$ can be represented by the union of $m+1$ disjoint connected symplectic surfaces $\Sigma_{g_{1}, \ldots, g_{m}} \amalg R_{X}$ or by a connected symplectic surface $\hat{\Sigma}_{g_{1}, \ldots, g_{m}}^{R_{X}}$ of genus $\sum_{i=1}^{m} g_{i}-m+1$ intersecting the (-2)-sphere $S_{R_{X}}$ in a single positive transverse point.

Then for any $g \geq 2$, there is a symplectic simply connected manifold $Y$ satisfying the following conditions:

(1) The canonical class $K_{Y}$ of $Y$ can be represented by the union $\Sigma_{g_{1}, \ldots, g_{m}, g}$ of $m+1$ disjoint connected symplectic surface of genus $g_{1}, \ldots, g_{m}, g$ or by a connected symplectic surface $\hat{\Sigma}_{g_{1}, \ldots, g_{m}, g}$ of genus $\sum_{i=1}^{m} g_{i}+g-m$.

(2) $Y$ contains a symplectic nucleus $N_{R_{Y}}$ with fiber $R_{Y}$ and section $S_{R_{Y}}$ disjoint from both $\Sigma_{g_{1}, \ldots, g_{m}, g}$ and $\hat{\Sigma}_{g_{1}, \ldots, g_{m}, g}$. The class $K_{Y}+\left[R_{Y}\right]$ can be represented by the union 
of $m+2$ disjoint connected symplectic surfaces $\Sigma_{g_{1}, \ldots, g_{m}, g} \amalg R_{Y}$ or by a connected symplectic surface $\hat{\Sigma}_{g_{1}, \ldots, g_{m}, g}^{R_{Y}}$ of genus $\sum_{i=1}^{m} g_{i}+g-m$ intersecting the (-2)-sphere $S_{R_{Y}}$ in a single positive transverse point.

Proof. Following [FS4], we observe that along the symplectic torus $R_{X} \subset X$ we can define the symplectic fiber sum

$$
X \#_{R_{X}=F} E(g)
$$

where $F$ is the standard fiber in $E(g)$. The resulting manifold is simply connected and symplectic, with canonical class $K_{X_{R_{X}}=F} E(g)=K_{X}+K_{E(g)}+2[F]=K_{X}+g[F]$. We have two symplectic representatives for that class. The first is the disjoint union $\Sigma_{g_{1}, \ldots, g_{m}} \amalg F_{1} \amalg \cdots \amalg F_{g}$, where the $F_{j}$ 's are parallel copies of $F$; this surface has $m+g$ connected components. For the second one, observing that the sum of 4.1) identifies $R_{X}$ and $F$, we can choose the disjoint union $\hat{\Sigma}_{g_{1}, \ldots, g_{m}}^{R_{X}} \amalg F_{2} \amalg \cdots \amalg F_{g}$; this surface has $g$ components. Figure 5 schematizes the situation for $X \#_{R_{X}=F} E(4)$.

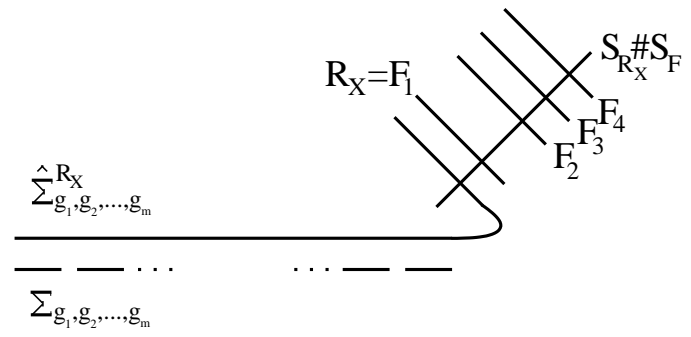

Fig. 5. Schematic presentation of the components of the two representatives of $K_{X}+g[F]$ (for $g=4)$; the first one is $\Sigma_{g_{1}, \ldots, g_{m}} \amalg \coprod_{i=1}^{4} F_{i}$ and the second one is $\hat{\Sigma}_{g_{1}, \ldots, g_{m}}^{R_{X}} \amalg \coprod_{i=2}^{4} F_{i}$.

As $g \geq 2$, the elliptic surface $E(g)$ of fiber $F$ contains a rim torus $R$ and a torus $T$ satisfying the conditions of Proposition 1 . The homology class $K_{X_{R_{X}=F} E(g)}+[R]=$ $K_{X}+g[F]+[R]$ is well defined and can be symplectically represented by the disjoint union $\Sigma_{g_{1}, \ldots, g_{m}} \amalg F_{1} \amalg \cdots \amalg F_{g} \amalg R$, with $m+g+1$ components, as well as by the disjoint union $\hat{\Sigma}_{g_{1}, \ldots, g_{m}}^{R_{X}} \amalg F_{2} \amalg \cdots \amalg F_{g-1} \amalg T$, with $g$ components. As in homology we have $[T]=[F]+[R]$, these two surfaces are homologous.

Next, we observe that the manifold $X \#_{R_{X}=F} E(g) \supset N_{R_{X}} \#_{R_{X}=F} E(g)$ contains a symplectically embedded configuration $\Gamma_{g}$, inherited from a configuration in $E(g)$, that intersects $F$ in a single point (its $-(g+2)$-sphere is the connected sum of $S_{R_{X}}$ and $S_{F}$ ). This configuration is disjoint from $\Sigma_{g_{1}, \ldots, g_{m}}$ (as the $(-2)$-sphere $S_{R_{X}}$ is disjoint from it) and from the symplectic nucleus with fiber $R$. Instead it does intersect the surface $\hat{\Sigma}_{g_{1}, \ldots, g_{m}}^{R_{X}}$ in a single positive transverse point in the $-(g+2)$-sphere. We blow down the configuration $\Gamma_{g}$. Denote by $Y$ the resulting simply connected, symplectic manifold. We point out that the nucleus $N_{R}$ survives the blowdown. 
The canonical class of $Y$ is given by the image of $K_{X \#_{R_{X}=F} E(g)}$ under the blowdown, and we will use the previous constructions to exhibit two symplectic representatives, one having $m+1$ connected components of genus $g_{1}, \ldots, g_{m}, g$, the other one connected and of genus $\sum_{i=1}^{m} g_{i}+g-m$.

First the disconnected representative $\Sigma_{g_{1}, \ldots, g_{m}, g}$ (presented in [FS4]) is obtained by the disjoint union of $\Sigma_{g_{1}, \ldots, g_{m}}$ (unaffected by the blowdown) and a genus $g$ connected surface $\hat{\Sigma}_{g}$ obtained, as in the initial step of our construction, by gluing the $g$ tori with hole, $\left(F_{1} \amalg \cdots \amalg F_{g}\right) \backslash v \Gamma_{g}$, to the sphere with $g$ holes, $S_{+} \cap B_{g}$. The connected representative $\hat{\Sigma}_{g_{1}, \ldots, g_{m}, g}$, instead, is obtained by gluing to $S_{+} \cap B_{g}$ the surface $\left(\hat{\Sigma}_{g_{1}, \ldots, g_{m}}^{R_{X}} \amalg\right.$ $\left.F_{2} \amalg \cdots U F_{g}\right) \backslash \nu \Gamma_{g}$. The resulting surface is clearly homologous to the previous one, and is connected, as both $F$ and $\hat{\Sigma}_{g_{1}, \ldots, g_{m}}^{R_{X}}$ intersect, in a positive transverse point, the $-(g+2)$-sphere of $\Gamma_{g}$. The genus of this surface is then easily computed. In reference to the scheme of Figure 5 the two representatives above are obtained by blowing down $\Gamma_{g}$, of which $S_{R_{X}} \# S_{F}$ is the first sphere, and connecting the surfaces hit by that sphere. This completes the proof of (1).

Next, we observe that the manifold $Y$ contains a symplectic nucleus, inherited from the one of $E(g)$, whose image we index by $R_{Y}$. This nucleus is disjoint, by construction, from both $\Sigma_{g_{1}, \ldots, g_{m}, g}$ and $\hat{\Sigma}_{g_{1}, \ldots, g_{m}, g}$. To finish our argument, we must proceed to identify a disconnected and a connected symplectic representative of the class $K_{Y}+\left[R_{Y}\right]$, as stated in (2). For what concerns the disconnected representative, this is simply provided by the disjoint union of the disconnected symplectic surface $\Sigma_{g_{1}, \ldots, g_{m}, g}$ and a copy of $R_{Y}$. In order to obtain the connected representative, we consider, in $X \#_{R_{X}=F} E(g)$, the surface $\hat{\Sigma}_{g_{1}, \ldots, g_{m}}^{R_{X}} \amalg F_{2} \amalg \cdots \amalg F_{g-1} \amalg T$, with $g$ components. By blowing down, as $\hat{\Sigma}_{g_{1}, \ldots, g_{m}}^{R_{X}}, F_{i}$ and $T$ intersect the configuration $\Gamma_{g}$ only in one point (on the sphere $S_{R_{X}} \# S_{F}$ ), the class $K_{Y}+\left[R_{Y}\right]$ has a symplectic connected representative $\hat{\Sigma}_{g_{1}, \ldots, g_{m}, g}^{R_{Y}}$ obtained by gluing, much as above, the genus $\sum_{i=1}^{m} g_{i}-m+1$ surface $\hat{\Sigma}_{g_{1}, \ldots, g_{m}}^{R_{X}}$ with one hole, and the $g-1$ tori with hole, $F_{2} \amalg \cdots \amalg F_{g-1} \amalg T$, to the sphere with $g$ holes, $S_{+} \cap B_{g}$. A check, using the genus formula for connected sum, or the adjunction formula, shows that the genus of the surface is the one stated. This surface intersects $S_{R_{Y}}$ in a single positive transverse point, which is the image of the intersection point of $T$ and $S_{R}$ under the blowdown.

Lemma 3 provides the inductive step required, and completes the construction of a symplectic connected surface, homologous to the disconnected symplectic representative of (1.1.

Acknowledgments. The author was supported in part by NSF grant \#0629956.

\section{References}

[ADK] Auroux, D., Donaldson, S., Katzarkov, L.: Luttinger surgery along Lagrangian tori and non-isotopy for singular symplectic plane curves. Math. Ann. 326, 185-203 (2003) Zbl 1026.57020 MR 1981618

[D] Donaldson, S.: Polynomials, vanishing cycles and Floer homology. In: Mathematics: Frontiers and Perspectives, Amer. Math. Soc., 55-64 (2001) Zbl 0958.57027 MR 1754767 
[DS] Donaldson, S., Smith, I.: Lefschetz pencils and the canonical class for symplectic 4manifolds. Topology 42, 743-785 (2003) Zbl 1012.57040 MR 1958528

[FS1] Fintushel, R., Stern, R.: Rational blowdowns of smooth 4-manifolds. J. Differential Geom. 46, 181-235 (1997) Zbl 0896.57022 MR 1484044

[FS2] Fintushel, R., Stern, R.: Knots, links and 4-manifolds. Invent. Math. 134, 363-400 (1998) Zbl 0914.57015 MR 1650308

[FS3] Fintushel, R., Stern, R.: Symplectic surfaces in a fixed homology class. J. Differential Geom. 52, 203-222 (1999) Zbl 0981.53085 MR 1758295

[FS4] Fintushel, R., Stern, R.: The canonical class of a symplectic 4-manifold. Turk. J. Math. 25, 137-145 (2001) Zbl 0977.57032 MR 1829084

[G] Gompf, R.: A new construction of symplectic manifolds. Ann. of Math. (2) 142, 527-595 (1995). Zbl 0849.53027 MR 1356781

[GM] Gompf, R., Mrowka, T.: Irreducible 4-manifolds need not be complex. Ann. of Math. (2) 138, 61-111 (1993) Zbl 0805.57012 MR 1230927

[GS] Gompf, R., Stipsicz, A.: 4-Manifolds and Kirby Calculus. Grad. Stud. Math. 20, Amer. Math. Soc. (1999) Zbl 0933.57020 MR 1707327

[S] Smith, I.: Symplectic submanifolds from surface fibrations. Pacific J. Math. 198, 197-206 (2001) Zbl 1062.53041 MR 1831978

[Sy] Symington, M.: Symplectic rational blowdown. J. Differential Geom. 50, 505-518 (1998) Zbl 0935.57035 MR 1852770

[T] Taubes, C.: Seiberg-Witten and Gromov Invariants for Symplectic Four-Manifolds. International Press (2000) Zbl 0967.57001 MR 1798809

[V] Vidussi, S.: Nonisotopic symplectic tori in the fiber class of elliptic surfaces. J. Symplectic Geom. 2, 207-218 (2004) Zbl 1078.57025 MR 2108374 